\title{
Growth inhibitory effect of adenovirus-mediated tissue-targeted expression of ribosomal protein L23 on human colorectal carcinoma cells
}

\author{
HENGHU FANG ${ }^{1}$, JINGBO KANG ${ }^{1}$, RUI DU ${ }^{1}$, XIANGFEI ZHAO ${ }^{1}$, XINHONG ZHANG $^{1}$, DONGQING REN ${ }^{2}$, \\ YAFEI ZHANG ${ }^{3}$, ZEJUN LU ${ }^{1}$, SHANSHAN WU ${ }^{1}$, WEI ZHENG ${ }^{1}$ and JUYI WEN ${ }^{1}$ \\ ${ }^{1}$ Department of Radiation Oncology, Navy General Hospital, Beijing; ${ }^{2}$ Department of Radiation Medicine and \\ The Ministry of Education, Key Laboratory of Hazard Assessment and Control in Special Operational Environment, \\ School of Public Health, Fourth Military Medical University, Xi'an; ${ }^{3}$ Department of Gastroenterology, \\ General Hospital of the Air Force, PLA, Beijing, P.R. China
}

Received March 15, 2015; Accepted May 6, 2015

DOI: $10.3892 /$ or.2015.4026

\begin{abstract}
A large body of evidence has established murine double minute 2 (MDM2) as a crucial negative regulator of p53 and the major suppressor of p53 function in tumors with wild-type (wt)-p53. Therefore, by inhibiting MDM2 one may reactivate $\mathrm{p} 53$ in tumor cells, leading to their demise. Previous studies revealed that ribosomal protein L23 (RPL23) inhibited MDM2-mediated p53 ubiquitination through direct binding to MDM2, and subsequently induced the p53 level as well as its activity, suggesting that it may be a candidate for use in tumor gene therapy. In the present study, we developed a recombinant adenoviral vector expressing the RPL23 gene under control of the carcinoembryonic antigen (CEA) promoter (rAd/CEA-RPL23), and using an in vitro system with cultured human colorectal carcinoma LoVo cells harboring the wt-p53 gene, we proved that $\mathrm{rAd} / \mathrm{CEA}-\mathrm{RPL} 23$ infection could induce the accumulation of endogenous wt-p53 protein and thus lead to the inhibition of tumor cell growth via inducing cell cycle arrest and apoptosis. In vivo treatment of rAd/CEA-RPL23 also exhibited a significant inhibitory effect on tumor growth in nude mice bearing LoVo xenografts. Furthermore, we showed that rAd/CEA-RPL23 synergized with classic chemotherapeutic agent 5-fluorouracil (5-FU) and enhanced its activity against LoVo cells in vivo and in vitro. Taken together, the data presented here suggest that CEA promoter-targeted exogenous RPL23 expression could be of therapeutic value against human colorectal carcinoma that retains wt-p53.
\end{abstract}

Correspondence to: Professor Jingbo Kang, Department of Radiation Oncology, Navy General Hospital, Beijing 100048, P.R. China E-mail: kjbnavy@163.com

Key words: ribosomal protein L23, p53, murine double minute 2, carcinoembryonic antigen, colorectal carcinoma

\section{Introduction}

The tumor-suppressor p53 is a potent transcription factor that controls a major pathway protecting cells from malignant transformation (1). As such, it is the most frequently inactivated protein in human tumors (2). The murine double minute 2 (MDM2) protein is one of the most well studied negative regulators of the p53 protein (3). It is currently known that MDM2 is overexpressed in a variety of human tumors, which results in the inactivation of wild-type (wt)-p53 protein, with an effect similar to that of mutations in the p53 gene $(4,5)$. Indeed, tumors with MDM2 gene amplification almost exclusively express wt-p53 (6). Therefore, reactivating p53 activity by antagonizing MDM2 offers a new therapeutic strategy in tumors that retain wt-p53 $(7,8)$.

Ribosomal protein L23 (RPL23) was discovered to be capable of inhibiting MDM2-mediated p53 ubiquitination through direct binding to MDM2, and subsequently induce the p53 level as well as its activity $(9,10)$. In addition, ectopically expressed RPL23 has been proved to be capable of interacting with MDM2 in both the nucleus and cytoplasm, which indicates that MDM2 is retained in the nucleus and cytoplasm as a complex, and this complex formation represents one more mechanism by which RPL23 indirectly inhibits MDM2-p53 binding (10). Therefore, there is a rationale for the use of RPL23 as a new target of gene therapy for tumors with wt-p53.

Many studies have shown that human colorectal carcinoma LoVo cells have the wt-p53 gene and the expressed p53 protein is functional and capable of inducing expression of the target genes (11-13). Therefore, LoVo cells are a suitable cell model with which to study the therapeutic utility of reactivating p53 in tumors with wt-p53. In the present study, we developed a recombinant adenoviral vector expressing the RPL23 gene under the control of the carcinoembryonic antigen (CEA) promoter (rAd/CEA-RPL23), and by using a LoVo cell model, we tested the therapeutic efficacy of RPL23 gene transfer on human colorectal carcinoma containing wt-p53 in vitro and in vivo. 


\section{Materials and methods}

Cell lines and reagents. Three cell lines were used in this study, including human colorectal cancer cell lines LoVo (wt-p53 gene) and HT29 (mutant p53 gene) (11), and normal human skin fibroblast (HSF) cells. All cells were maintained in RPMI-1640 supplemented with 10\% FCS (Invitrogen Corp., Carlsbad, CA, USA), at $37^{\circ} \mathrm{C}$, in a humidified atmosphere containing $5 \% \mathrm{CO}_{2}$. Mouse monoclonal antibodies specific for p53 (DO-1), p21 and PUMA were obtained from Santa Cruz Biotechnology Inc. (Santa Cruz, CA, USA), and mouse monoclonal $\beta$-actin antibody was obtained from Sigma-Aldrich Chemical Co. (St. Louis, MO, USA). The ApoAlert ${ }^{\mathrm{TM}}$ CPP32/Caspase-3 Assay kit was purchased from Clontech Laboratories Inc. (Palo Alto, CA, USA). All reagents were used in different concentrations as indicated.

Construction of the recombinant adenovirus. Human CEA promoter fragment was obtained by PCR from human genomic DNA (primers: forward, 5'-GAAGATCTAGAGCATGGGGA GACCCG-3' and reverse, 5'-ATTTGCGGCCGCTCTGTGG AGAAGAGCTTG-3'), and cloned into the shuttle plasmid pAdTrack resulting in pAdTrack-CEAp. The full-length of human RPL23 cDNA with a 6-his tag was cut from pcDNA3.1-RPL23 constructed by our laboratory, and subcloned into pAdTrack-CEAp resulting in pAdtrack-CEAp-RPL23 which was linearized by $P m e I$ and transformed into AdEasier-1 cells. Transformants were selected on LB agar plates containing $25 \mu \mathrm{g} / \mathrm{ml}$ kanamycin, and positive pAdEasy-CEAp-RPL23 was identified by electrophoretic analysis of the PCR products (primers: forward, 5'-CTGCTGGGTTTCTCTGTCACA AAG-3' and reverse, 5'-ATGGTGATGGTGATGATGTGC AAT-3'), then digested with $P a c$, and transfected into 293 cells to produce the recombinant adenovirus Ad/CEA-RPL23 (rAd/CEA-RPL23). RT-PCR analysis was performed to determine the specific expression of RPL23 gene transcription driven by the CEA promoter in human colorectal cancer LoVo and HT29 cells (primers: forward, 5'-GCCGCGATATCATGG GCATGT-3' and reverse, 5'-GGTGATGGTGATGATGTGC AAT-3'). rAd/CEA-lacZ was constructed by a similar method. The adenoviral transduction efficiency of the three tested cells was evaluated by flow cytometry (Epics XL; Coulter, Miami, FL, USA) using adenovirus-expressing green fluorescent protein (GFP) (rAd5-GFP) alone (14).

Assay of cell growth. Cells were plated in triplicate wells into a 96-well plate at a density of $5 \times 10^{3}$ cells $/ 100 \mu \mathrm{l}$, and cultured routinely for $24 \mathrm{~h}$. The supernatant was discarded, and infected with recombinant adenoviruses at various multiplicity of infection (MOI) for $24 \mathrm{~h}$, and remained in culture with RPMI-1640 supplemented with $10 \%$ FCS. Four days after adenovirus infection, cell growth was assessed by 3-(4,5-dimethylthiazol-2-yl)-2,5-diphenyltetrazolium bromide (MTT) assay (15).

Cell cycle analysis. LoVo cells cultured in 6-well plates (Corning) were infected with the recombinant adenoviruses at various MOI for $48 \mathrm{~h}$, and then were harvested by centrifugation. After washing with ice-cold PBS, the cells were suspended in $\sim 0.5 \mathrm{ml}$ of $70 \%$ alcohol and kept at $4^{\circ} \mathrm{C}$ for $30 \mathrm{~min}$. The suspension was filtered through $50-\mu \mathrm{m}$ nylon mesh, and the DNA content of stained nuclei was analyzed by a flow cytometer (Epics XL; Coulter). The cell cycle was analyzed using Multicycle-DNA Cell Cycle Analyzed software.

Detection of apoptosis. Detection of apoptotic cells by flow cytometry was performed as described previously (16). LoVo cells cultured in 6-well plates (Corning) were infected with recombinant adenoviruses at various MOI for $48 \mathrm{~h}$ before cells were harvested, and then Annexin V/propidium iodide binding assay was performed by a flow cytometer (Epics XL; Coulter).

Western blot analysis. LoVo cells infected with recombinant adenoviruses at various MOI for $48 \mathrm{~h}$ were collected, washed twice in cold PBS, and lysed at $4^{\circ} \mathrm{C}$ in lysis buffer using protease and phosphatase inhibitors as described previously (15). Cell lysates containing $50 \mu \mathrm{g}$ total protein were resolved in $10 \%$ SDS-PAGE, transferred to nitrocellulose polyvinylidene difluoride membranes (Amersham Pharmacia Biotech, Piscataway, NJ, USA), and probed with primary antibodies. Detection and signal visualization were conducted using appropriate secondary antibodies conjugated with horseradish peroxidase (Santa Cruz Biotechnology Inc.) and enhanced chemiluminescence reagents (Pierce, Rockford, IL, USA) according to the manufacturer's instructions.

5-Fluorouracil (5-FU) sensitivity of tumor cells infected with rAd/CEA-RPL23. LoVo cells were seeded in triplicate in 96-well plates at a density of $5 \times 10^{3}$ cells/well in $100 \mu \mathrm{l}$ of medium. Twenty-four hours later, the cells were infected with rAd/CEA-RPL23 (or rAd/CEA-lacZ) at an MOI of 50. After another $24 \mathrm{~h}$ of incubation, the medium was changed to fresh medium containing $1 \mu \mathrm{mol} / 15$-FU. The cells were then cultured for an additional $24 \mathrm{~h}$ before being harvested for apoptotic analysis or caspase-3 activity analysis.

Measurement of caspase-3 activity. Caspase-3 activity was determined according to the user's manual for the ApoAlert ${ }^{\mathrm{TM}}$ CPP32/Caspase-3 Assay kit. The supernatant obtained by a centrifugation of lysed cells was added to the reaction mixture containing dithiothreitol and caspase-3 substrate (N-acetyl-Asp-Glu-Val-Asp-p-nitroanilide) and incubated for $1 \mathrm{~h}$ at $37^{\circ} \mathrm{C}$. Absorbance of the chromophore p-nitroanilide produced was measured at $405 \mathrm{~nm}$. The standard curves were obtained from the absorbance of p-nitroanilide standard reagent diluted with cell lysis buffer (up to $20 \mathrm{nM}$ ). One unit of the enzyme was defined as the activity producing $1 \mathrm{nmol}$ of p-nitroanilide.

In vivo experiments using animal models. Male Balb/c athymic nude mice (6- to 8-weeks of age) (Shanghai Laboratory Animal Center of the Chinese Academy of Sciences) were used for the experiments, as approved by the Institutional Animal Care and Use Committee at the Southwest Hospital. In addition, the experiments were conducted according to guidelines for the Welfare of Animals in Experimental Neoplasia published by The United Kingdom Coordinating Committee on Cancer Research.

A mouse subcutaneous tumor model was established as described previously (17). LoVo cells $\left(1 \times 10^{6}\right.$ in PBS/100 $\left.\mu \mathrm{l}\right)$ 
A

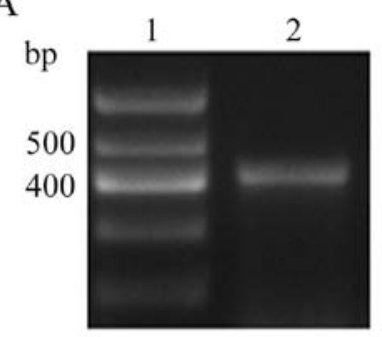

B

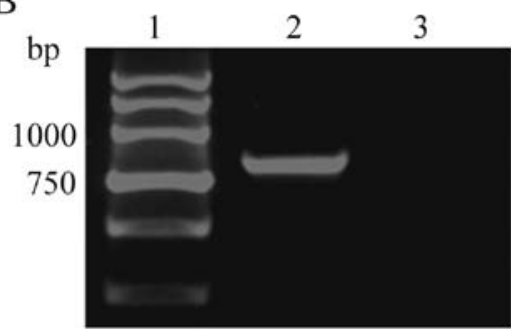

$\mathrm{C}$

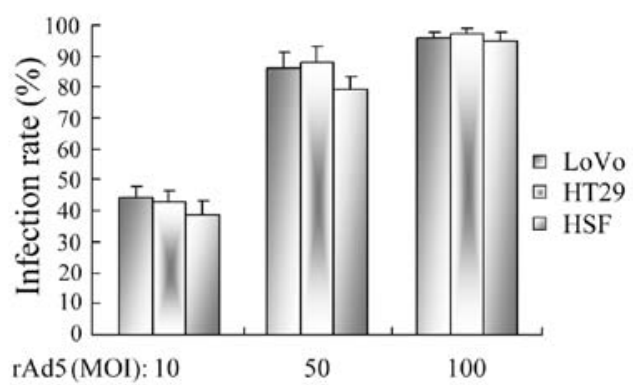

$\mathrm{D}$

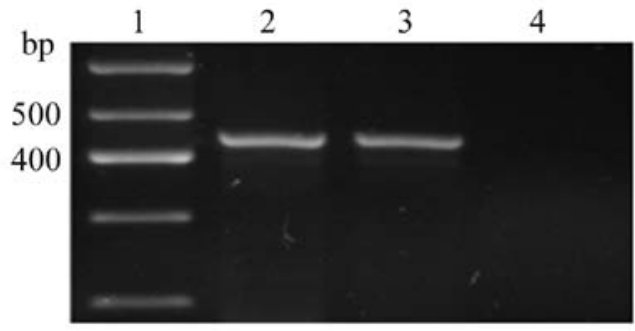

Figure 1. Construction and identification of rAd/CEA-RPL23. (A) CEA promoter fragment amplified by PCR (414 bp). Lane 1, DNA marker; lane 2, CEA promoter. (B) Target segment in pAdEasy-CEAp-RPL23 amplified by PCR (823 bp). Lane 1, DNA marker; lane 2, pAdEasy-CEAp-RPL23; lane 3, pAdEasy. (C) Infection rate of recombinant adenovirus. The efficiency of adenovirus (rAd5) infection into tested cells was evaluated through flow cytometry assay. When the MOI was set at 50, the infection rate of the recombinant adenovirus in the LoVo, HT29 and HSF cells was $86.3 \pm 4.9,88.2 \pm 5.1$ and $79.4 \pm 4.1 \%$, respectively, and at MOI 100 the rate was $>95 \%$ in all the three cell lines $(n=4, P<0.05)$. (D) RT-PCR products of the RPL23 gene transcripts in cells infected with rAd/CEA-RPL23 (447 bp). Lane 1, DNA marker; lane 2, LoVo; lane 3, HT29; lane 4, HSF. CEA, carcinoembryonic antigen; RPL23, ribosomal protein L23; MOI, multiplicity of infection; HSF, human skin fibroblasts; rAd5, recombinant adenovirus serotype 5.

were injected subcutaneously into the right flanks of nude mice. Forty days after tumor cell inoculation, the mice were divided randomly into 3 groups (5 mice/group) and were treated every 3 days for a total of 5 times by way of multiple-center intratumor injection of $\mathrm{rAd} / \mathrm{CEA}-\mathrm{RPL} 23$ or $\mathrm{rAd} / \mathrm{CEA}-\mathrm{lacZ}$ at $5 \times 10^{7}$ plaque forming units (pfu) $/ 50 \mu 1$ per animal or PBS as a blank control. Serial changes in tumor volume were estimated every 3 days after the start of the recombinant adenovirus treatment. The volume of the tumors was calculated according to the formula: Tumor volume $=\left(\right.$ length $\mathrm{x}$ width $\left.{ }^{2}\right) / 2$.

In order to evaluate the therapeutic efficacy of 5-FU combined with rAd/CEA-RPL23 on disseminated colorectal carcinoma, a nude mouse model was established by intraperitoneal inoculation of $2 \times 10^{6}$ LoVo cells (18). Seven days later, the mice were divided randomly into the following 5 groups (10 mice/group) according to treatment schedules: a, injection of PBS as blank control; b, injection of 5-FU alone; $c$, injection of rAd/CEA-RPL23 alone; d, injection of rAd/CEA-RPL23 followed by $5-\mathrm{FU}$; and e, injection of $\mathrm{rAd} / \mathrm{CEA}-\mathrm{lacZ}$ followed by 5 -FU. In groups c, d, and e, the adenovirus $\left(2 \times 10^{8} \mathrm{pfu} / 200 \mu \mathrm{l}\right)$ was injected into mice via tail vein at days $7,10,13,16$ and 19 after the intraperitoneal inoculation of LoVo cells. In all groups, 5 -FU (500 $\mathrm{mg} / \mathrm{kg}$ body weight) or PBS was administered to mice via tail vein injection one day after the adenovirus injection. Survival of the animals was observed daily.

Statistical analysis. Quantitative results are expressed as the mean \pm SE. Statistical analysis was performed by using ANOVA and the LSD t-test. The survival rates were estimated using the Kaplan-Meier method, and the differences were analyzed by using the log-rank test to compare the resulting curves of the treatment groups. $\mathrm{P}<0.05$ was considered as statistically significant. All statistical analyses were performed using SPSS14.0 software (SPSS Inc., Chicago, IL, USA).

\section{Results}

Construction of rAd/CEA-RPL23 and the CEA-dependent RPL23 gene expression in human colorectal cancer cells. Researchers have found that the CEA promoter specifically promotes the exogenous gene expression in CEA-positive tumor cells, for which it has been extensively used in the target gene therapy of tumors (19-21). In the present study, we obtained the CEA promoter fragment by PCR (Fig. 1A), and using it, we constructed a new replication-deficient recombinant adenovirus, termed rAd/CEA-RPL23, containing the RPL23 gene under control of the CEA promoter (Fig. 1B). Through flow cytometry assay, the efficiency of adenovirus infection was evaluated by counting the percentage of GFP-positive cells after rAd-GFP infection, and as shown in Fig. 1C, among the LoVo, HT29 and HSF cells, there was no significant difference in the infection rate of the recombinant adenovirus serotype 5 (rAd5) when the MOI was set at 10,50 or 100 . Furthermore, by RT-PCR analysis, we determined that upon $\mathrm{rAd} / \mathrm{CEA}-\mathrm{RPL} 23$ infection at the same dose (50 MOI), the RPL23 gene product was expressed specifically in the CEA-positive human colorectal cancer LoVo and HT29 cells (17,22), but not in the CEA-negative HSF cells (Fig. 1D).

Growth inhibitory effect of $r A d / C E A-R P L 23$ on colorectal cancer cells. To show that the growth inhibitory effect of $\mathrm{rAd} / \mathrm{CEA}-\mathrm{RPL} 23$ is dependent on p53 gene status in colorectal cancer cells, wt-p53 LoVo and mutant-p53 HT29 colorectal carcinoma cell lines were infected with increasing MOI of the 

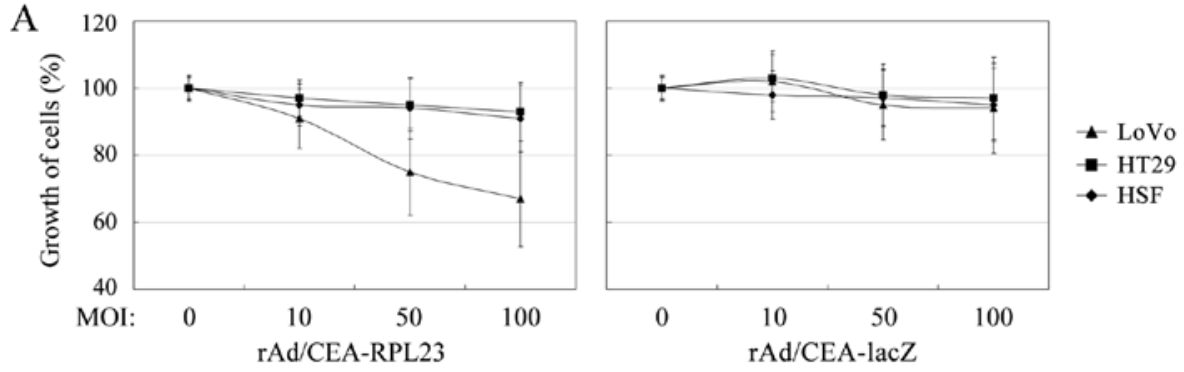

\section{B}

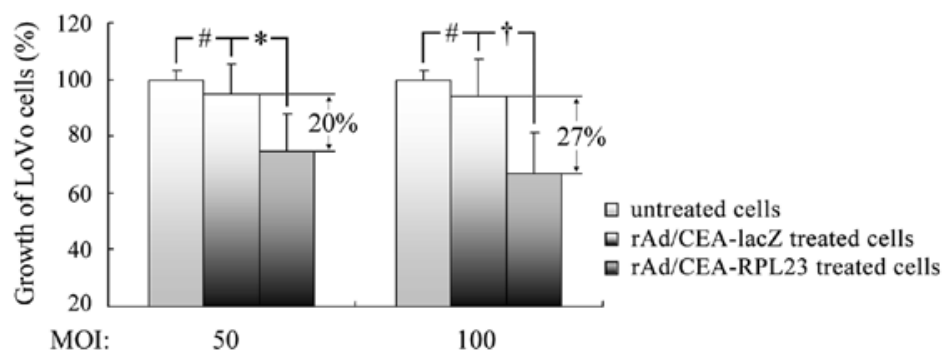

Figure 2. Growth inhibitory effect of rAd/CEA-RPL23 on colorectal cancer cells. Cell growth was evaluated by MTT assay as described in Materials and methods. The cell growth of blank control cells (untreated cells) was determined to be 1 (100\%), and correspondingly, the cell growth of the treated cells was calculated according to the following formula: Cell growth = absorbance of treated cells/absorbance of blank control cells $\mathrm{x} 100 \%$. (A) A dose-dependent inhibition of cell growth was exhibited in the LoVo but not in the HT29 and HSF cells treated with the same MOI of rAd/CEA-RPL23. No significant effect was observed in the cells treated with rAd/CEA-lacZ. (B) The growth inhibitory effect of rAd/CEA-RPL23 on LoVo cells. The results are presented as mean (SD) from four independent experiments. ${ }^{*} \mathrm{P}>0.05,{ }^{*} \mathrm{P}<0.05$ and ${ }^{\dagger} \mathrm{P}<0.01$. CEA, carcinoembryonic antigen; RPL23, ribosomal protein L23; HSF, human skin fibroblasts.

recombinant adenovirus. Through MTT assay, a dose-dependent growth inhibitory activity of rAd/CEA-RPL23 infection was revealed in the LoVo but not in the HT29 cells (Fig. 2A). As depicted in Fig. 2B, compared with control rAd/CEA-lacZ, $\mathrm{rAd} / \mathrm{CEA}-\mathrm{RPL} 23$ infection at MOI 50 and 100 decreased the growth of viable LoVo cells by 20 and $27 \%$, respectively. In regards to the growth of HT29 cells with mutant p53 and CEA-negative HSF cells, rAd/CEA-RPL23 treatment did not differ from the rAd/CEA-lacZ treatment (Fig. 2A, P>0.05).

rAd/CEA-RPL23 induces cell cycle arrest in LoVo cells through stabilization of 553 and upregulation of p21. The above data demonstrated that rAd/CEA-RPL23 infection significantly inhibited the growth of LoVo cells in vitro. To determine whether rAd/CEA-RPL23 induces endogenous p53 accumulation and thus consequent cell cycle arrest in LoVo cells, flow cytometric assay and western blot analysis were performed. As shown in Fig. 3A, $48 \mathrm{~h}$ after infection with rAd/CEA-RPL23 at MOI 10, 50 and 100, the fraction of LoVo cells in the G1 phase was increased approximately by 5.4, 34.2 and $52.5 \%$, respectively, associated with a concentration-dependent decrease in the $\mathrm{S}$ phase fraction, indicating cell cycle arrest at the G1-S checkpoint. As expected, in LoVo cells infected with $\mathrm{rAd} / \mathrm{CEA}-\mathrm{lacZ}$, no significant cell cycle arrest was observed $(\mathrm{P}>0.05)$. Western blot analysis further revealed that the level of endogenous p53 protein in LoVo cells was increased upon rAd/CEA-RPL23 infection, indicating that adenovirus-mediated RPL23 expression could induce stabilization and accumulation of wt-p53 protein through inhibiting MDM2-p53 interaction (Fig. 3C). A parallel increase in the expression of cyclin-dependent kinase inhibitor p21, the known transcriptional target of p53 (23), was found in LoVo cells upon rAd/CEA-RPL23 infection (Fig. 3C), suggesting that the accumulation of wt-p53 protein induced by $\mathrm{rAd} / \mathrm{CEA}-\mathrm{RPL} 23$ was functional and capable of mediating downstream signal transduction.

rAd/CEA-RPL23 induces apoptosis in LoVo cells through activation of the p53 apoptotic pathway. Since we also observed significant cell death in LoVo cells upon rAd/CEA-RPL23 infection, we next assessed the apoptosis using Annexin V-propidium iodide staining and flow cytometry. As shown in Fig. 3B, treatment of LoVo cells with $\mathrm{rAd} / \mathrm{CEA}-\mathrm{RPL} 23$ for $48 \mathrm{~h}$ at 50 and $100 \mathrm{MOI}$ resulted in 7.5 and $16.1 \%$ apoptotic cell death, respectively $(\mathrm{P}<0.05)$, and under similar conditions, rAd/CEA-lacZ did not show the ability to induce apoptosis $(\mathrm{P}>0.05)$. By western blot analysis, we further revealed that the expression level of pro-apoptotic protein PUMA, a known transcriptional target of p53 (24), was increased in the LoVo cells upon rAd/CEA-RPL23 infection (Fig. 3C), suggested that the accumulation of wt-p53 protein induced by rAd/CEA-RPL23 was also capable of triggering the p53 apoptotic pathway.

Intratumoral injection of rAd/CEA-RPL23 suppresses the growth of established LoVo subcutaneous tumors in nude mice. To test the potential utility of RPL23 gene therapy in the treatment of human colorectal carcinoma, we tested the efficacy of rAd/CEA-RPL23 in vivo using a mouse subcutaneous tumor model. One week after inoculation of LoVo cells, rAd/CEA-RPL23, rAd/CEA-lacZ or PBS was administered locally by way of multiple-center intratumor injection. As shown in Fig. 4, the LoVo tumor growth was significantly suppressed in the mice given treatment of rAd/CEA-RPL23 as compared to that in the control groups $(\mathrm{P}<0.05)$. The size 
A

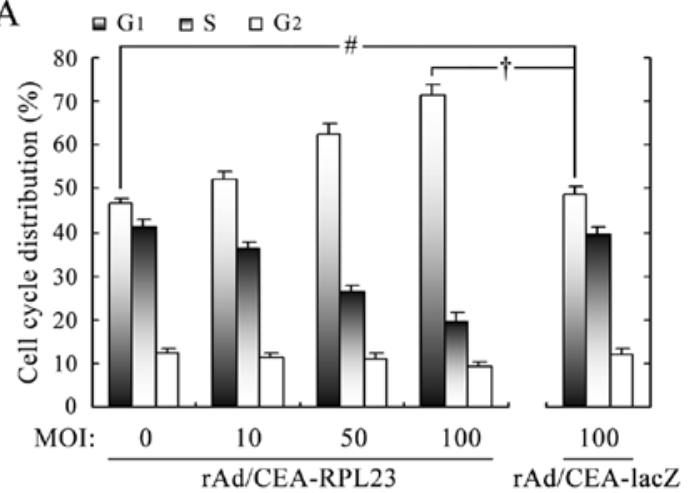

B

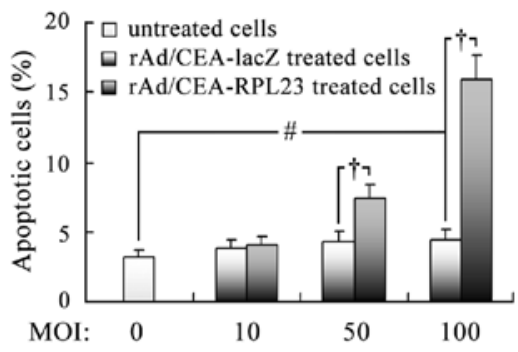

$\mathrm{C}$ MOI: $\quad$\begin{tabular}{cccc} 
& rAd/CEA-RPL23 & rAd/CEA-lacZ \\
\cline { 2 - 4 } & 10 & 50 & 100
\end{tabular}

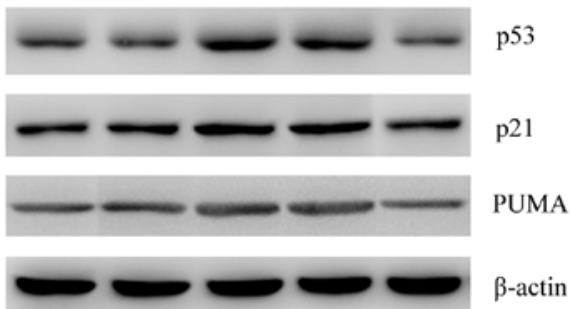

Figure 3. Cell cycle distribution and apoptosis analysis of LoVo cells upon rAd/CEA-RPL23 infection. Forty-eight hours after treatment of recombinant adenoviruses at various MOI, the cells were collected for flow cytometric analysis. (A) Cell cycle distribution of LoVo cells. (B) Apoptosis of LoVo cells. The results are presented as mean (SD) from four independent experiments. ${ }^{\prime \prime} \mathrm{P}>0.05,{ }^{\dagger} \mathrm{P}<0.01$. (C) Western blot analysis revealed that rAd/CEA-RPL23 infection resulted in accumulation of wt-p53 protein and subsequently led to increased expression levels of $\mathrm{p} 21$ and PUMA. $\beta$-actin was used as an internal control. Similar results were obtained in another two independent experiments. CEA, carcinoembryonic antigen; RPL23, ribosomal protein L23; MOI, multiplicity of infection; wt, wild-type.

A

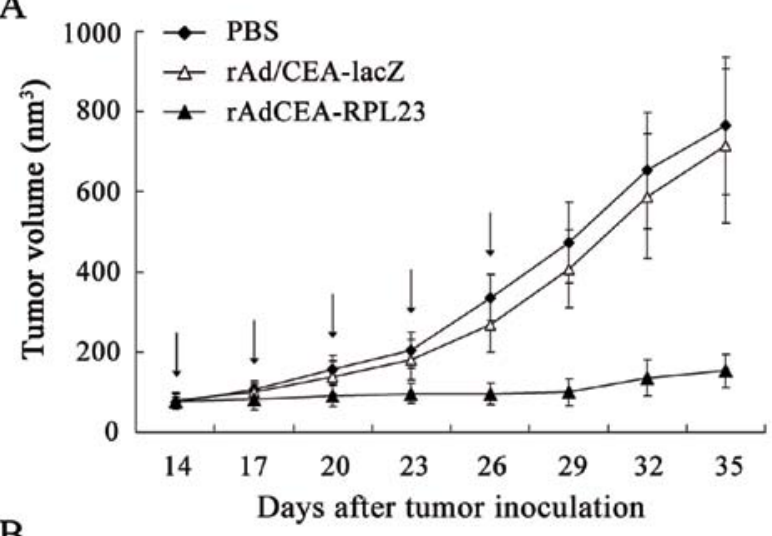

B

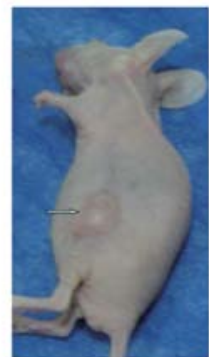

Treatment: PBS

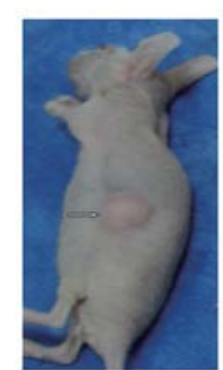

rAd/CEA-lacZ rAd/CEA-RPL23

Figure 4. Suppression of LoVo tumor growth by intratumoral injection of rAd/CEA-RPL23. Subcutaneous tumors derived from LoVo cells were treated with rAd/CEA-RPL23 or the controls as described in Materials and methods. (A) Tumor volume was monitored over time after the start of treatment. Arrows indicate the time-points when treatment was adminstered. Values represent means $\pm \mathrm{SD}(\mathrm{n}=5)$. The effect of the treatment with $\mathrm{rAd} / \mathrm{CEA}-\mathrm{RPL} 23 \mathrm{differed}$ significantly from other control groups $(\mathrm{P}<0.05)$. (B) Representative images of a mouse in each group at the end of the experiment were shown. CEA, carcinoembryonic antigen; RPL23, ribosomal proteins L23. 

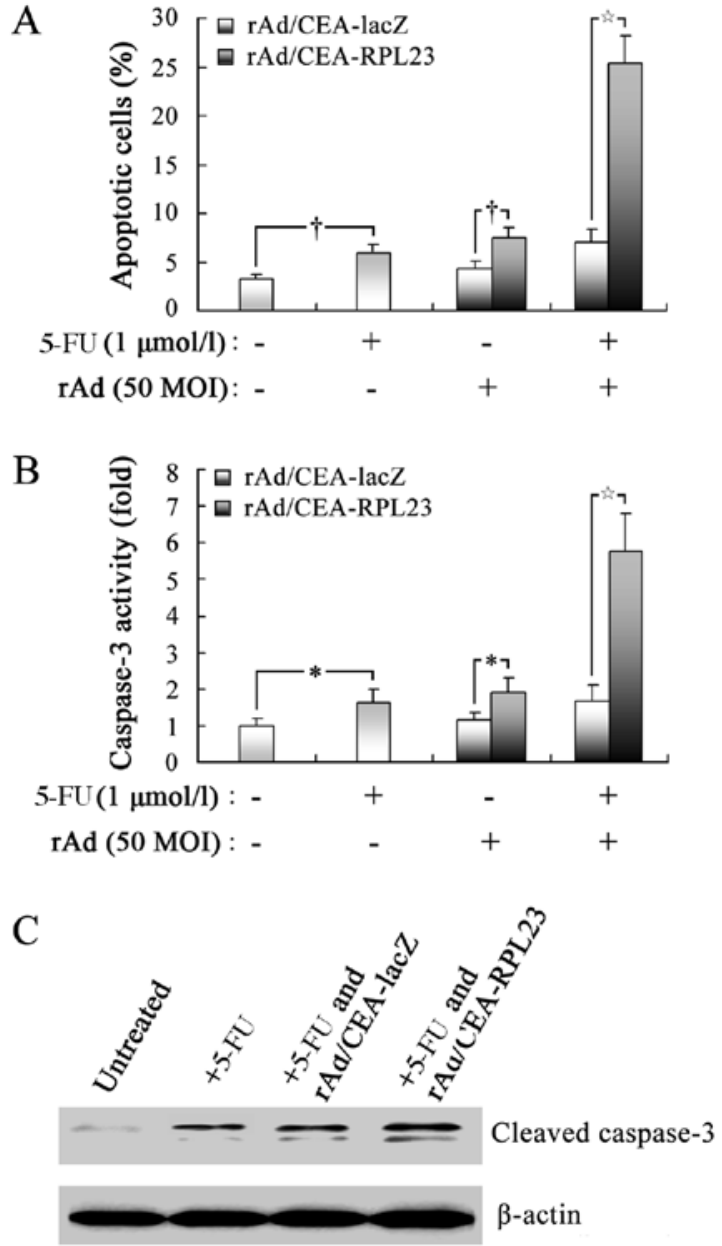

Figure 5. Effect of rAd/CEA-RPL23 on the sensitivity of LoVo cells to 5-FU-induced apoptosis. LoVo cells were treated with 5-FU alone or 5-FU after $24 \mathrm{~h}$ pretreatment with rAd/CEA-RPL23, and then the cells were harvested for apoptosis and caspase-3 activity analysis as described in Materials and methods. (A) Apoptotic rate of LoVo cells. (B) Caspase-3 activity of LoVo cells. The results are presented as mean (SD) from four independent experiments. ${ }^{\top} \mathrm{P}<0.01$, ${ }^{*} \mathrm{P}<0.001$ and ${ }^{*} \mathrm{P}<0.05$. (C) Western blot analysis was further used to examine the expression of cleaved caspase- 3 in LoVo cells upon treatment with 5-FU alone or 5-FU combined with rAd/CEA-RPL23. $\beta$-actin was used as an internal control. Similar results were obtained in other two independent experiments. CEA, carcinoembryonic antigen; RPL23, ribosomal protein L23; 5-FU, 5 -fluorouracil.

of the tumors between the rAd/CEA-RPL23 group and the rAd/CEA-lacZ or PBS group was significant different from day 20 through the experiment period $(\mathrm{P}<0.05)$. The difference between the rAd/CEA-lacZ and PBS group was not statistically significant $(\mathrm{P}>0.05)$.

Stabilization of wt-p53 protein upon rAd/CEA-RPL23 infection enhances 5-FU-induced apoptosis in LoVo cells. Research has shown that loss of p53 gene function in colorectal carcinoma cells abolishes the apoptotic response to 5-FU (25). In the present study, combined treatment of LoVo cells with a relatively low dose of rAd/CEA-RPL23 and 5-FU was performed to evaluate whether stabilization of wt-p53 protein upon $\mathrm{rAd} / \mathrm{CEA}$-RPL23 infection could enhance 5-FU-induced apoptosis. Although single agent 5 -FU at $1 \mu \mathrm{mol} / 1$ was able to induce apoptosis in LoVo cells, the apoptosis induced by treatment in combination with rAd/CEA-RPL23 at MOI 50

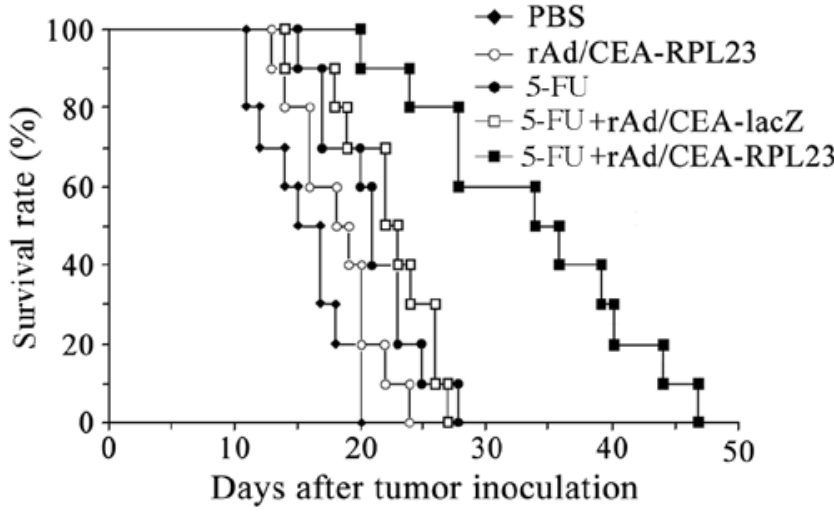

Figure 6. Therapeutic effect of 5-FU combined with rAd/CEA-RPL23 on survival periods in a disseminated colorectal carcinoma model. The nude mice which were intraperitoneally inoculated with LoVo cells $\left(2 \times 10^{6}\right.$ cells/mouse $)$ were randomly divided into the following 5 groups (10 mice/group) according to treatment schedules: group a, $\bullet$, injection of PBS as blank control; b, o, injection of rAd/CEA-RPL23 alone; c, $\bullet$, injection of 5-FU alone; d, $\square$, injection of rAd/CEA-lacZ followed by 5-FU; e, $\mathbf{n}$, injection of rAd/CEA-RPL23 followed by 5-FU. Survival of animals was observed everyday. 5-FU, 5-fluorouracil; CEA, carcinoembryonic antigen; RPL23, ribosomal protein L23.

was significantly greater than that following treatment of 5-FU alone. As shown in Fig. 5A, the apoptotic rate in the 5-FU alone group and rAd/CEA-RPL23 alone group was 6.0 and $7.5 \%$, respectively, while, the apoptotic rate in the combined treatment group was $25.3 \%$, which was more than the additive effect of each treatment separately $(25.3 \%$ vs. $6.0+7.5 \%)$. These results were confirmed by caspase-3 activity assay. Caspase-3 is a central executioner of apoptosis (26). In the present study, it was found that the caspase- 3 activity of LoVo cells treated with the combination of $50 \mathrm{MOI} \mathrm{rAd} / \mathrm{CEA}-\mathrm{RPL} 23$ and $1 \mu \mathrm{mol} / 1$ 5-FU was increased significantly to 5.78 -fold when compared with the control, whereas the activity following treatment with single 5-FU or rAd/CEA-RPL23 was only increased slightly to 1.64- and 1.91-fold of the control, respectively (Fig. 5B). Western blot analysis gave similar result, which showed that upon combination treatment with 5-FU and rAd/CEA-RPL23, the expression level of cleaved caspase-3 was markedly increased as compared to the levels following treatment of 5-FU or rAd/CEA-RPL23 alone (Fig. 5C).

rAd/CEA-RPL23 improves the therapeutic efficacy of 5-FU in a murine model with disseminated LoVo tumors. To evaluate the therapeutic efficacy of 5-FU combined with $\mathrm{rAd} / \mathrm{CEA}-\mathrm{RPL} 23$ in vivo, a murine model with intraperitoneally disseminated LoVo tumors that closely resemble human advanced colorectal carcinoma was designed. Fig. 6 shows the survival rates of animals after the inoculation of LoVo cells. The median survival time of the mice treated with 5-FU combined with $\mathrm{rAd} / \mathrm{CEA}-\mathrm{RPL} 23$ ( $39 \pm 3.3$ days) was longer than that of the mice treated with 5 -FU alone ( $25 \pm 3.8$ days) or 5-FU combined with rAd/CEA-lacZ ( $23 \pm 3.0$ days). Log-rank test revealed that the survival was significantly longer for mice in the 5-FU+rAd/CEA-RPL23 group as compared with that in the 5-FU group or 5-FU+rAd/CEA-lacZ group $(\mathrm{P}<0.05)$. Additionally, treatment of rAd/CEA-RPL23 alone also showed a detectable therapeutic effect on the survival of mice as compared with the blank control $(19 \pm 1.3$ days vs. 
$15 \pm 2.0$ days), although the difference was not statistically significant $(\mathrm{P}>0.05)$.

\section{Discussion}

Since overexpression or hyperactivation of MDM2 contributes to functional inactivation of wt-p53 in nearly $50 \%$ of tumors (27), and since MDM2 is capable of attenuating the effectiveness of chemotherapies that induce p53 (28), antagonizing MDM2 is being considered an attractive strategy for the treatment of tumors (5,7). Different approaches have been exploited in this strategy including inhibition of MDM2 expression, MDM2 ubiquitin ligase activity and MDM2-p53 binding (7). Recently, a small-molecule compound termed Nutlin-3a, which specifically inhibited the MDM2-p53 interaction and thus led to stabilization of p53 and activation of the p53 pathway, was discovered and has been reported to display a potent growth-inhibitory effect on a variety of tumor cells that contain wt-p53 $(7,29)$. The $\mathrm{p} 14^{\mathrm{ARF}}$ protein, encoded by the INK4a/ARF locus, was found to bind to MDM2 directly and inhibit the ubiquitin E3 ligase function of MDM2. It has been reported that the $\mathrm{p} 14^{\mathrm{ARF}}$ gene transfer mediated by adenovirus vector inhibited the growth and enhanced the response of chemotherapies in a variety of wt-p53 tumor cells (30-32). RPL23 is another cellular protein with the capacity of binding to MDM2 and inhibiting the ubiquitin E3 ligase function of MDM2 $(9,10)$, and Jin et al reported that the ability of RPL23 to inhibit MDM2's E3 ligase function was comparable to that of p14 ${ }^{\mathrm{ARF}}(9)$, suggesting a rationale for using RPL23 as a new target for tumor gene therapy.

The therapeutic utility of reactivating p53 depends on two critical factors: defective p53 signaling in tumor cells with wt-p53 may attenuate or disable the therapeutic response; and possible growth suppressive and/or apoptotic activity of p53 in normal tissues may narrow or eliminate the therapeutic window (7). Human colorectal carcinoma LoVo cells have been confirmed to express functional p53 protein (11-13). Therefore, in the present study, LoVo cells were used as a model system to evaluate the therapeutic efficacy of RPL23 gene transfer on human tumors with wt-p53. The other key factor regarding the therapeutic utility of pharmacological p53 activation is p53 toxicity to normal tissues. Since embryonic genes such as CEA are usually transcriptionally silent in adult normal tissues but overexpressed in malignant tumor, the use of an embryonic gene promoter to regulate expression of a target gene for the purpose of reducing the toxicity of gene transfer into normal tissues is popular (19-21). In the present study, we constructed a recombinant adenoviral vector expressing the RPL23 gene driven by the CEA promoter (rAd/CEA-RPL23). In theory, rAd/CEA-RPL23 can deliver the RPL23 gene specifically to colorectal cancer cells but not to normal tissue cells, by which, rAd/CEA-RPL23 infection is expected to be slightly toxic to normal tissues.

Through flow cytometry and western blot analysis, we validated that the exogenous RPL23 protein expression by adenoviral vector under control of the CEA promoter was restricted to CEA-positive LoVo and HT29 human colorectal carcinoma cells but not CEA-negative HSF cells. Furthermore, we determined that the exogenous RPL23 protein could stabilize endogenous p53 protein and thus induce cell cycle arrest and apoptosis in wt-p53 LoVo cells. An in vivo study showed that the growth of subcutaneous tumors derived from LoVo cells was significantly inhibited by direct intratumor injection of rAd/CEA-RPL23. Our study also discovered that rAd/ CEA-RPL23 infection had no obvious effect on the growth of p53-mutant HT29 human colorectal cancer cells, which suggested that the growth inhibiting effect of exogenous RPL23 expression was mainly via p53-dependent mechanisms.

5 -FU is a widely used chemotherapeutic drug for the treatment of human colorectal carcinoma. The antitumor activity of 5-FU has been attributed in part to its ability to induce p53-dependent cell growth arrest and apoptosis $(33,34)$. Since hyperactivation of MDM2 has been proved to be capable of attenuating 5-FU-induced toxicity by inhibiting p53 activation (35), we reasoned that a combination treatment of 5-FU and RPL23 gene transfer may improve the prognosis of patients with colorectal carcinoma after surgery. The results of our in vitro experiment showed that rAd/CEA-RPL23 pretreatment in LoVo cells enhanced 5-FU-induced apoptosis, and assay of the in vivo therapeutic efficacy revealed that the combination treatment increased the overall survival of mice with disseminated LoVo tumors. These results indicate that RPL23 gene transfer may be used as part of a combined therapy for the treatment of human tumors. Notably, the combination treatment also showed a detectable enhancement on the survival of mice with disseminated HT29 tumors (data not shown), indicating that RPL23 gene transfer may influence the sensitivity of tumor cells to antitumor drugs via a p53-independent manner. As it has been determined that ectopically expressed RPL23 is capable of retaining MDM2 both in the cytoplasm and the nucleus (10), we hypothesized that the exogenous RPL23 expression may improve 5-FU efficacy in tumors with mutant p53 via its function of blocking MDM2 nuclear translocation. For example, MDM2 is essential to the activation of $\mathrm{NF}-\kappa \mathrm{B}$ in some tumor cells $(36,37)$, and therefore, the blocking of MDM2 nuclear translocation by ectopically expressed RPL23 may lead to a reduction in $\mathrm{NF}-\kappa \mathrm{B}$ activation, which has been proved to directly contribute to chemotherapy resistance and metastasis in human tumors (38). Therefore, suppression of the $\mathrm{NF}-\kappa \mathrm{B}$ pathway may be another mechanism by which RPL23 gene transfer exhibits a synergistic effect with antitumor drugs such as 5-FU, regardless of the p53 status.

In conclusion, the present study demonstrated that exogenous RPL23 expression driven by the CEA promoter may be of therapeutic value against human colorectal carcinoma that retains wt-p53.

\section{Acknowledgements}

The present study was supported by the China Postdoctoral Science Foundation (no. 20100481468).

\section{References}

1. Selivanova G: Wild type p53 reactivation: from lab bench to clinic. FEBS Lett 588: 2628-2638, 2014.

2. Khoo KH, Verma CS and Lane DP: Drugging the p53 pathway: understanding the route to clinical efficacy. Nat Rev Drug Discov 13: 217-236, 2014.

3. Kruse JP and Gu W: Modes of p53 regulation. Cell 137: 609-622, 2009.

4. Freedman DA, Wu L and Levine AJ: Functions of the MDM2 oncoprotein. Cell Mol Life Sci 55: 96-107, 1999. 
5. Wang W and El-Deiry WS: Restoration of p53 to limit tumor growth. Curr Opin Oncol 20: 90-96, 2008.

6. Momand J, Jung D, Wilczynski S and Niland J: The MDM2 gene amplification database. Nucleic Acids Res 26: 3453-3459, 1998.

7. Zhao Y, Aguilar A, Bernard D and Wang S: Small-molecule inhibitors of the MDM2-p53 protein-protein interaction (MDM2 inhibitors) in clinical trials for cancer treatment. J Med Chem 58: 1038-1052, 2015.

8. Lu C and El-Deiry WS: Targeting p53 for enhanced radio- and chemo-sensitivity. Apoptosis 14: 597-606, 2009.

9. Dai MS, Zeng SX, Jin Y, Sun XX, David L and Lu H: Ribosomal protein L23 activates p53 by inhibiting MDM2 function in response to ribosomal perturbation but not to translation inhibition. Mol Cell Biol 24: 7654-7668, 2004.

10. Jin A, Itahana K, O'Keefe K and Zhang Y: Inhibition of HDM2 and activation of p53 by ribosomal protein L23. Mol Cell Biol 24 7669-7680, 2004.

11. Pocard M, Chevillard S, Villaudy J, Poupon MF, Dutrillaux B and Remvikos Y: Different $\mathrm{p} 53$ mutations produce distinct effects on the ability of colon carcinoma cells to become blocked at the G1/S boundary after irradiation. Oncogene 12: 875-882, 1996.

12. Shi MD, Lin HH, Lee YC, Chao JK, Lin RA and Chen JH: Inhibition of cell-cycle progression in human colorectal carcinoma Lovo cells by andrographolide. Chem Biol Interact 174: 201-210, 2008.

13. Zhang NH, Song LB, Wu XJ, Li RP, Zeng MS, Zhu XF, Wan DS Liu Q, Zeng YX and Zhang XS: Proteasome inhibitor MG-132 modifies coxsackie and adenovirus receptor expression in colon cancer cell line Lovo. Cell Cycle 7: 925-933, 2008.

14. Kim HJ, Cho HI, Han YH, Park SY, Kim DW, Lee DG, Kim JH, Shin WS, Paik SY, Kim CC, et al: Efficient transduction with recombinant adenovirus in EBV-transformed B lymphoblastoid cell lines. J Biochem Mol Biol 37: 376-382, 2004.

15. Zhang Y, Shi Y, Li X, Du R, Luo G, Xia L, Du W, Chen B, Zhai H, Wu K, et al: Proteasome inhibitor MG132 reverses multidrug resistance of gastric cancer through enhancing apoptosis and inhibiting P-gp. Cancer Biol Ther 7: 540-546, 2008.

16. Li X, Zhang Y, Xiong C, Jin H, Jing B, Zhang Y and Fan D: Overexpression of a new gene P28GANK confers multidrug resistance of gastric cancer cells. Cancer Invest 27: 129-139, 2009.

17. Li Y, Chen Y, Dilley J, Arroyo T, Ko D, Working P and Yu DC: Carcinoembryonic antigen-producing cell-specific oncolytic adenovirus, OV798, for colorectal cancer therapy. Mol Cancer Ther 2: 1003-1009, 2003.

18. Cao G, Kuriyama S, Gao J, Kikukawa M, Cui L, Nakatani T, Zhang X, Tsujinoue H, Pan X, Fukui H, et al: Effective and safe gene therapy for colorectal carcinoma using the cytosine deaminase gene directed by the carcinoembryonic antigen promoter. Gene Ther 6: 83-90, 1999.

19. Schrewe H, Thompson J, Bona M, Hefta LJ, Maruya A Hassauer M, Shively JE, von Kleist S and Zimmermann W: Cloning of the complete gene for carcinoembryonic antigen: analysis of its promoter indicates a region conveying cell type-specific expression. Mol Cell Biol 10: 2738-2748, 1990

20. Qiao J, Doubrovin M, Sauter BV, Huang Y, Guo ZS, Balatoni J, Akhurst T, Blasberg RG, Tjuvajev JG, Chen $\mathrm{SH}$, et al: Tumor-specific transcriptional targeting of suicide gene therapy. Gene Ther 9: 168-175, 2002.
21. Ueda K, Iwahashi M, Nakamori M, Nakamura M, Matsuura I, Ojima T and Yamaue H: Improvement of carcinoembryonic antigen-specific prodrug gene therapy for experimental colon cancer. Surgery 133: 309-317, 2003.

22. Dabrowska A, Szary J, Kowalczuk M, Szala S and Ugorski M: CEA-negative glioblastoma and melanoma cells are sensitive to cytosine deaminase/5-fluorocytosine therapy directed by the carcinoembryonic antigen promoter. Acta Biochim Pol 51: 723-732, 2004.

23. Chen X, Bargonetti J and Prives C: p53,through p21 (WAF1/CIP1), induces cyclin D1 synthesis. Cancer Res 55: 4257-4263, 1995.

24. Yu J and Zhang L: No PUMA, no death: implications for p53-dependent apoptosis. Cancer Cell 4: 248-249, 2003.

25. Sobrero A, Kerr D, Glimelius B, Van Cutsem E, Milano G, Pritchard DM, Rougier P and Aapro M: New directions in the treatment of colorectal cancer: a look to the future. Eur J Cancer 36: 559-566, 2000.

26. Kuribayashi K, Mayes PA and El-Deiry WS: What are caspases 3 and 7 doing upstream of the mitochondria? Cancer Biol Ther 5: 763-765, 2006

27. Vogelstein B, Lane D and Levine AJ: Surfing the p53 network. Nature 408: 307-310, 2000.

28. Rayburn E, Zhang R, He J and Wang H: MDM2 and human malignancies: expression, clinical pathology, prognostic markers, and implications for chemotherapy. Curr Cancer Drug Targets 5: 27-41, 2005.

29. Shangary S and Wang S: Small-molecule inhibitors of the MDM2-p53 protein-protein interaction to reactivate $\mathrm{p} 53$ function: a novel approach for cancer therapy. Annu Rev Pharmacol Toxicol 49: 223-241, 2009.

30. Wang W, Rastinejad F and El-Deiry WS: Restoring p53-dependent tumor suppression. Cancer Biol Ther 2 (Suppl 1): S55-S63, 2003.

31. Deng X, Kim M, Vandier D, Jung YJ, Rikiyama T, Sgagias MK, Goldsmith M and Cowan KH: Recombinant adenovirus-mediated p14(ARF) overexpression sensitizes human breast cancer cells to cisplatin. Biochem Biophys Res Commun 296: 792-798, 2002.

32. Hemmati PG, Gillissen B, von Haefen C, Wendt J, Stärck L, Güner D, Dörken B and Daniel PT: Adenovirus-mediated overexpression of p14(ARF) induces $\mathrm{p} 53$ and Bax-independent apoptosis. Oncogene 21: 3149-3161, 2002.

33. Kaeser MD, Pebernard S and Iggo RD: Regulation of p53 stability and function in HCT116 colon cancer cells. J Biol Chem 279: 7598-7605, 2004

34. Sun XX, Dai MS and Lu H: 5-fluorouracil activation of p53 involves an MDM2-ribosomal protein interaction. J Biol Chem 282: 8052-8059, 2007.

35. Wang H, Oliver P, Zhang Z, Agrawal S and Zhang R: Chemosensitization and radiosensitization of human cancer by antisense anti-MDM2 oligonucleotides: in vitro and in vivo activities and mechanisms. Ann NY Acad Sci 1002: 217-235, 2003.

36. Gu L, Findley HW and Zhou M: MDM2 induces NF-kappaB/p65 expression transcriptionally through Sp1-binding sites: a novel, p53-independent role of MDM2 in doxorubicin resistance in acute lymphoblastic leukemia. Blood 99: 3367-3375, 2002.

37. Cheney MD, McKenzie PP, Volk EL, Fan L and Harris LC: MDM2 displays differential activities dependent upon the activation status of NFkappaB. Cancer Biol Ther 7: 38-44, 2008.

38. Shen HM and Tergaonkar V: NFkappaB signaling in carcinogenesis and as a potential molecular target for cancer therapy. Apoptosis 14: 348-363, 2009. 